\title{
Criminologie
}

\section{Les victimes de viol devant les tribunaux à la fin du Moyen Âge d'après les sources dijonnaises et lyonnaises}

\section{Nicole Gonthier}

Volume 27, numéro 2, 1994

Sexe et criminalité

URI : https://id.erudit.org/iderudit/017353ar

DOI : https://doi.org/10.7202/017353ar

Aller au sommaire du numéro

\section{Éditeur(s)}

Les Presses de l'Université de Montréal

ISSN

0316-0041 (imprimé)

1492-1367 (numérique)

Découvrir la revue

Citer cet article

Gonthier, N. (1994). Les victimes de viol devant les tribunaux à la fin du Moyen Âge d'après les sources dijonnaises et lyonnaises. Criminologie, 27(2), 9-32.

https://doi.org/10.7202/017353ar
Résumé de l'article

At the end of the Middle Ages, the manner in which the courts of justice punished rapists clearly shows how seriously such crimes were judged at that time. Endeavoring to increase their juristic power and worrying that the propagation of rape would corrupt the entire society and threaten its survival, impelled the judges and their officials - princes, bishops, and municipal bodies - to deal with all complaints and to initiate inquiries about them. A study of the progressive steps undertaken in the preliminary investigations of these cases reveals that after an inquiry into the accuser's reputation, the judges demanded numerous proofs from the victim about the rape. Moreover, a clinical examination was demanded from a midwife. Thus, in addition to the scientific proof and the witness's statements, the investigators were able to arrive at a better assessment of the rapist. The rapist, in turn, under the distressful solitude in his cell where he had been incarcerated by the police as soon as the complaint was made, finally admitted to the crime and renounced having degraded and defamed his accuser, $\mathrm{ft}$ was then up to the court to punish the offender. These offenders were treated severely, particularly if they had attacked moral and social values by deflowering children and virgins. 


\section{LES VICTIMES DE VIOL DEVANT LES TRIBUNAUX À LA FIN DU MOYEN ÂGE D'APRÈS LES SOURCES DIJONNAISES ET LYONNAISES Nicole Gonthier'}

At the end of the Middle Ages, the manner in which the courts of justice punished rapists clearly shows how seriously such crimes were judged at that time. Endeavoring to increase their juristic power and worrying that the propagation of rape would corrupt the entire society and threaten its survival, impelled the judges and their officials - princes, bishops, and municipal bodies - to deal with all complaints and to initiate inquiries about them. A study of the progressive steps undertaken in the preliminary investigations of these cases reveals that after an inquiry into the accuser's reputation, the judges demanded numerous proofs from the victim about the rape. Moreover, a clinical examination was demanded from a midwife. Thus, in addition to the scientific proof and the witness's statements, the investigators were able to arrive at a better assessment of the rapist. The rapist, in turn, under the distressful solitude in his cell where he had been incarcerated by the police as soon as the complaint was made, finally admitted to the crime and renounced having degraded and defamed his ac. cuser. It was then up to the court to punish the offender. These offenders were treated severely, particularly if they had attacked moral and social values by deflowering children and virgins.

Parmi les crimes qui relèvent de la haute justice médiévale, parmi ceux qui demeurent longtemps au rang des «cas royaux» ou des causes retenues par le dêtenteur du ban quand celui-ci a concédé des pouvoirs de justice à des vassaux ou à des magistrats urbains, figure le crime de rapt ou de viol.

Ce degré de gravité ne doit pas tout au droit romain mais prend aussi ses sources dans le droit germanique, puisque les codes de lois proclamaient déjà auprès des peuples francs. burgondes ou wisigoths le domınage extrême cuusé par un tel acte. Le viol, en effet. combine le vol et la violence, et l'ampleur de ses conséquences n'affecte pas seulement la victime mais ses proches. sa lignée. sa descendance, dont il compromet la pureté et la légitimité. Dès le haut Moyen Âge. un tel acte a valu à ceux qui s'en rendaient coupables des sanctions exemplaires et rigoureuses, paiement d'un wergeld énorme, mutilations invalidantes, voire mise à mort. La gravité de ce crime continue à être affirmée par les coutumiers au XIIl ${ }^{\mathbb{C}}$ siècle,

1. Prolesseur J'histoire médiévale à l'Université Jcan-Moulin-Lyon Ill. 
par les législations royale, seigneuriales ou municipales, à la fin du Moyen Âge.

La fréquence de ce type de criminalité semble pourtant s“intensifier aux XIV et $X V^{\mathfrak{e}}$ siècles. On a avancé. pour expliquer ce phénomène, de multiples raisons outre le fait que l'on dispose de sources plus nombreuses et plus détaillées. Le viol des femmes ${ }^{2}$ en temps de guerre n'est pas l'apanage des époques barbares. Alors qu'il s'applique encore de nos jours à l'occasion de certains conflits et dans des contrées que l'on croyait arrivées à un haut degré de civilisation, il sévit particulièrement dans le royaume de France, parcouru par les armées du roi d'Angleterre et par les routiers de tout poil en raison de la guerre de Cent Ans, il se déchaîne avec les haines de la guerre civile des Armagnacs et des Bourguignons, il reste l'un des maux redoutés de la soldatesque recrutée pour les guerres d'Italie, sous les règnes de Charles VIII et de Louis XII.

Toutefois, les violeurs ne se comptent pas seulement chez les routiers et les mercenaires. Jacques Rossiaud a donné des viols collectifs nombreux. répertoriés dans quelques villes de France, une analyse tout autre, mettant en cause le déséquilibre du sex-ratio, le sureffectif des jeunes mâles par rapport au nombre des filles à marier ${ }^{3}$. Il est vrai que l'ampleur du développement économique de certains centres urbains en fait des pôles attractits pour une population de célibataires à la recherche d'un emploi: les villes universitaires regroupent, elles aussi, une proportion de clercs de moins de trente ans qui contribue à ce déséquilibre et rend le risque des viols plus marqué. Enfïn. le retard apporté au mariage des fils, par des pères soucieux de garder longtemps l'autorité suprême sur l'ouvroir. la parenté et la domesticité. provoquerait cette déviation du désir sexuel où s'exercent tout à la fois la recherche d'une jouissance physique et la volonté de démontrer sa supériorité sur un être plus faible, que la soumission avilit.

La clef de ce problème de société doit également être recherchée dans le courant antiféministe qu'exprime assez la littérature de cette époque. Dans le Décameron, dans les Fabliaux et quelques romans satiriques, la femme est dépeinte sous les traits d'une débauchée capable des pires tromperies pour satisfaire sa soif de plaisirs, ou comme une harpie qu'il faut

2. Dans Ies pages qui suivent, on n'envisagera que les viols s'appliquant aux personnes de sexe féminin. En effet, quelques très rares cas de viols ou de tentatives de viols homosexucls viennent devant la justice mais alors le crime de sodomic. considéré comme un péché abominable, emporte seul l'attention des juges et le partenaire, entaché du mêne crime, finit par être condamné aussi. D'où le peu de zèle des victimes éventuelles à dénoncer pareil forfait!

3. Rossiaud, J., (1988), La Prostifution médiévale, Paris, Flammarion, pp. 26-40 qui reprennent les conclusions de son article des Annales ESC, 1976, $\mathbf{n}^{\circ} 2$, pp. 289-325, intitulé : "Prostitution, jeunesse et société au XV" siècle». 
soumettre à moins de n'avoir plus d'honneur dans son ménage, tandis que le Roman de la Rose exalte l'abandon de toute créature humaine à la loi de nature, sans restriction. Une ère de permissivité sexuelle semble s'ouvrir aux pires moments de ces deux siècles, quand le spectacle de la mort noire. fulgurante et massive, fait comprendre combien la vie est courte, quand l'encadrement dogmatique. ralenti et discrédité par les contradictions du Grand Schisme, ne soutient plus assez la réflexion morale des chrétiens.

Il faudrait sans doute ajouter à ces facteurs qui rendent la tentation du viol plus obsédante que jamais. certaines contingences de la vie quotidienne telle la médiocrité de l'habitat, qui exagère la promiscuité de l'occupation. le manque d'intimité. le «voyeurisme» du voisinage dont tant de documents judiciaires nous donnent la preuve, tandis que la fragilité des matériaux rend souvent bien aléatoire le rempart d'une porte ou d'une fenêtre fermées ${ }^{4}$. Ajoutons enfin que les crimes de viol nous sont mieux connus en ces temps de la fin du Moyen Âge grâce aux modifications de la procédure. Alors que jusqu'au milieu du XIII ${ }^{e}$ siècle, il fallait une plainte formée pour que s'ouvre un procès (procédure accusatoire), aux XIVe et $\mathrm{XV}^{\mathrm{e}}$ siècles, les cours laïques, suivant en cela l'exemple des tribunaux ecclésiastiques qui ont «expérimenté » la procédure inquisitoire contre les hérétiques cathares, adoptent le principe de la saisie sur «commune renommée 5 .

Il ne s'agit pas ici de reprendre l'étude déjà entreprise par A. Porteau-

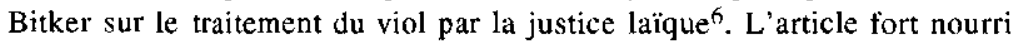
que cet auteur consacre au sujet repose sur les sources coutumières du nord de la France, principalement, ainsi que sur les fonds du Parlement de Paris et du Trésor des chartes. Se limitant. vu l'ampleur de l'enquête. aux XIIIe et XIVe siècles, il analyse le crime de viol et sa résolution judiciaire en privilégiant l'agresseur et les modes de répression que les justices laïques peuvent lui appliquer. Dans les pages qui suivent on essaiera plutôt de donner la parole aux victimes du viol, en utilisant des sources plus tardives, celles du $X V^{c}$ siècle, et en choisissant, parmi le corpus des documents judiciaires, les pièces très précieuses que sont les extraits d'instruction. Aux Archives de la Côte d'Or. à Dijon, existe un fonds très volumineux de pièces de ce type. correspondant aux affaires traitées par le tribunal échevinal de la capitale des ducs de Bourgogne ${ }^{7}$. Depuis le XII ${ }^{\mathrm{e}}$ siècle en effet. les échevins de

4. Cf. Gonthier, N., (1992), Cris de haine et rites d'unité, la violence dans les ville (XII' ${ }^{\mathrm{e}}-\mathrm{XVI}^{\mathrm{e}}$ siècles), Brépols, Turnhout, pp. 87-97.

5. Laingui, A. ct Lebigre, A., Histoire du droit pénal, t. $11:$ La Procédure criminelle, Cujas, Paris, pp. 55-57.

6. A. Porteau-Bitker, «La justice laĭque ct le viol au Moyen Âge», dans Revue historique de droit français et étranger, 1988, pp. 491-526.

7. Il s'agit de la série BII 360 qui couvre les procès de la ville (instruction, sentences), de 1386 à 1503, soit 17 volumes de liasses. À ce fonds, dejà ample, słajoute celui des causes de la mairie et des causes de l'hôtel des séries BII 336 et BII 337. 
Dijon disposent d'un droit de haute et basse justice qui leur a été concédé par le duc Hugues IIl, dans la charte de commune de 1183-1187. Ils l'exercent avec application. dans le souci de ne pas laisser au prince l'occasion de contisquer ce droit sous prétexte de désordre public. Toute instruction menée consigne donc les interrogatoires de la victime, des principaux tếmoins et de l'accusé. On en saisit le déroulement chronologique, puisque chaque pièce est datée: on peut également apprécier les réactions spontanees des participants, les «coups de théâtre » intervenant parfois au fil des questions, dans un texte dont la forme juridique se rompt alors pour laisser retentir la parole populaire, retranscrite en langage vernaculaire. C'est ce genre de source. si appréciable pour sa vivacité. que les archives lyonnaises fournissent également, d'une manière plus parcimonieuse, il est vrai. Point de justice consulaire, ici, mais une justice seigneuriale de l'archevêque de Lyon s'exerçant sur la ville, ainsi qu'une justice seigneuriale des chanoines cathédraux dans leurs diverses obéances ${ }^{8}$. Là encore. la parole des victimes ou des accusés se déploie, retranscrite dans la langue franco-provençale, au sein du texte de l'instruction, généralement en Jatin.

Nous essaierons donc de tirer le meilleur parti d'une telle documentation afin de déterminer comment ce crime de viol est reçu, traité, et sanctionné par les tribunaux et de découvrir en vertu de quels critères sociaux. moraux ou politiques, les jugements en la matière se fondent.

\section{L'OUVERTURE DE L'ENQUÊTE JUDICIAIRE}

\section{Des plaintes timides}

On a vu que pour intervenir, la justice n'a plus besoin d'une plainte formée. Une simple dénonciation, une diffamation ou une rumeur suffisent.

Cependant, les victimes hésitent à parler de l'attentat qu'elles ont subi $^{9}$. On comprend mieux ce comportement lorsque l'on découvre quelle sorte de personnes est le plus souvent la proie des violeurs. Il s'agit de très jeunes filles, placées comme servantes. Elles ont quitté le foyer familial et ne bénéficient plus de la protection paternelle. À partir de leur entrée en service, toutefois, le devoir de tutelle et d'éducation que remplissaient leurs parents incombe en principe à leurs maîtres, tout comme l'artisan prend en charge la formation professionnelle et morale d'un apprenti.

8. Obéance, de obedientia, terme lyonnais désignant l'ensemble des droits et des revenus appartenant à un chapitre sur une paroisse ou un groupe de paroisses. Les revenus d'une même obéance étaient partagés entre plusieurs obéanciers dont le plus important, le mansionnaire, avait seul les droits de justice.

9. Sur l'analyse de ce numerus obscurus, cf. l'analyse de J. Rossiaud, dans La Prostitution médiévale, chapitre II, pp. 26-28, à partir des registres des ADCO, BIl 360 qu il a largement utilisés. 
On voit d'ailleurs certains de ces maîtres s'entremettre auprès de la justice au nom de leur servante, la soutenir dans une démarche qui sans doute lui paraît trop compliquée ou trop humiliante. Ainsi, Jehan Morel, maître orfèvre, dont la servante. Jehannote, a été ravie par une troupe de compagnons, consent à appuyer de son autorité la plainte portée par la mère de la victime auprès du mayeur de Dijon ${ }^{10}$. Dans cette même ville, c'est par le biais de François le Clerc, le médecin qui l'employait, que le rapt de Pernotte, sa servante, est connu de la cour échevinale. Tout en avouant sa couardise, qui lui a interdit d'empêcher l'attentat («doubtant estre battuz, oultragers par lesd. Il compaignons de guerre [...] il n'eust oncques se grant paour»), il retrouve énergie et volonté devant le clerc de la cour pour dênoncer «le cas qui est grant oultrage et dont il se plaingt à justice [...] regardant sus ce estre faite bonne justice ainsi qu'il appartiendra par raison ${ }^{14}{ }$.

Plus souvent, la fillette se confie aux femmes de la maison. Employée dans l'hôtel du riche boucher Thévenin Pitoul, la petite Catherine, âgée de dix ans, qu'un valet a déflorée, vient se plaindre à la maîtresse de la maisonnée, en présence de la nourrice et de la chambelière de celle-ci ${ }^{12}$.

La solitude de ces victimes paraît cependant assez tragique : elles subissent les pressions de la famille du violeur qui leur intime le silence et se fait complice de l'agression par souci d'honorabilité. Catherine ne reçoit aucun secours de la femme Thévenin qui s'efforce de faire disparaître les traces de l'outrage mais ordonne à la fillette «qu'elle n'en dist mot à son pere ne a sa mere». Quant à Marguerite. servante de quinze ans, dont son maitre «fait son plaisir» en profitant de sa naïveté, elle risque une première allusion à ce qui lui est arrivé auprès de quelques femmes du voisinage. lesquelles se chargent de transmettre l'information à l'épouse trompée. Mais - mentionne Marguerite dans sa déposition - «elle n'en a tenu compte», aussi la victime "plus avant n'en a ozé parler ${ }^{13}$ ».

La publication du crime pourrait ainsi échapper à la justice, s'il n'y avait d'autres relais pour le divulguer. Le plus fréquent demeure le bavardage des femmes, de celles qui recueillent le honteux secret des victimes ou qui interviennent pour faire obstacle à l'agresseur. La servante d'un notaire. Richote, se soustrait aux assauts de son maître en criant «tresfort par plusieurs fois» et tellement qu'elle porte le délit sur la place publique. la voisine, alertée, ne se contente pas de frapper a l'huis jusqu'à ce qu'on lui ouvre, elle couvre le coupable de reproches injurieux : «Sanglant conchies,

10. Archives départementales de Côte d'Or, BII $360 \% 1, \mathrm{n}^{\circ} 8$, pièce $\mathrm{n}^{\circ} 3,22$ avril 1431, affaire Jehannote de Vauls.

11. ADCO, BII $360 / 11,10$ janvier 1467 , affaire Pernotle.

12. ADCO, BII 360/01, pièce $n^{\circ} 16,12$ mars 1418, affaire Catherine Malonne.

13. ADCO, BII 360/04, $\mathrm{n}^{\circ}$ 1, pièce 358,22 mai 1449, aff aire Margucrite Guillène. 
liares, bornes, putier, pourquoy est tu volu deshonorer ceste fille». Le voisinage s'émeut de la querelle dont le niveau sonore ne cesse d'amplifier, puis des femmes emmènent Richote, pleurante et dolente. La plainte que vient produire Richote, le 12 juin 1432, sans doute sur le conseil de ces «proudes femmes» semble presque superflue, le scandale public, la rumeur auraient suffi à déclencher le processus de l'enquête.

Ce sont encore les femmes qui prennent en charge le sort de la jeune Perrotte violée par un valet. Aux cris de l'enfant. elles accourent armées de fourches, viennent à bout du violeur et le conduisent elles-mêmes aux prisons, appliquant la procédure du tTagrant délit ${ }^{14}$.

\section{La plainte formée}

Si l'enquête a commencé sans que la victime en soit l'instigatrice par une plainte formée, une telle plainte semble cependant nécessaire pour que les coupables puissent être confondus. Ainsi, quand le procureur de Dijon, après moult «descentes de police» dans plusieurs hôtels de la ville, retrouve enfin, en une chambre de la rue de la Chaudronnerie, la servante qui fut ravie de force par une bande armée, il déclare avoir «incontinent demandé [à celle-ci] selle se plaignoit point dudit Audrieu, laquelle [lui] a répondu que oy». Elle décline d'ailleurs, à la suite de ce oui, tout le texte de l'accusation, résumant les chefs d'inculpation qui seront ceux du procès.

Même si elle paraît humiliante car elle oblige - nous le verrons plus loin - à des dépositions qui choquent la pudeur des femmes, un certain nombre de victimes osent prononcer cette plainte personnelle. Elle témoigne d'une confiance en la justice, conçue comme protectrice de ceux qu'abandonnent les alliances traditionnelles de la parenté. Les plus humbles filles sont ainsi entendues et leurs dires pris en consideration. Cette jeune femme qui se loue à la journée pour travailler dans les vignes de Dijon et qui ne trouve aucun secours contre les assauts de son employeur parmi les personnes présentes se tourne vers le tribunal «soy complaignant ${ }^{15}$ ». Il faut du courage pour porter une plainte redoutée par les coupables. Ces derniers cherchent à intimider leur victime, multiplient les menaces, ne reculent pas devant les voies de fait: de tels obstacles se dressent sur le chemin de Marguerite Bargain, la fille du trompette de Dijon, que «certains compaignons poursuyvaient pour fere pechié et qui la menoissoient de battre». S'étant fait placer en «l'especiale saulve garde du Roy», elle a déjà reçu le renfort de la justice contre ses agresseurs mais il lui faut encore essuyer de nombreux coups, le jour où «elle s'en al (a) plaindre à monseigneur le mayeur de Dijon». Rien n'arrête cependant ce caractère bien trempé, elle

14. ADCO, BII 360/03, $\mathrm{n}^{\circ} 242,6$ juillet 1444.

15. ADCO, BII $360 / 08$, piece $n^{\circ} 29,3$ juin 1462, affaire Nicole, épouse de Richard Blaise. 
ose braver les violents en proclamant «qu'elle s'en aloit plaindre au procureur du Roy ${ }^{16}$ ».

La menace de la plainte en justice est brandie par la victime tandis que le viol est perpétré. Elle se mêle aux cris d'appel au secours, la femme hésitant entre les pleurs et les paroles de vengeance. Enlevée au lit de son mari par un groupe de trois coquins et forcée plusieurs fois par chacun d'eux. Gillote affirme qu'elle "crioit tresfort qu'elle s'en plaindroit en justice» puis elle ajoute que lorsqu'ils «eurent ainsi fait leurs plaisirs d'elle, ilz la laisserent aler»... mais «elle leur dist qu'elle les feroit pandre ${ }^{17}$ ».

Cette association entre dénonciation et vengeance démontre combien, lorsqu'elle porte plainte, la femme sait qu'une machine redoutable va se mettre en branle et quelle confïance elle garde en une action implacable de la justice. L'accusation de viol, prise avec un tel sérieux par les juges, peut donc bien devenir une arme entre les mains des femmes. Sur les allégations d'une jeune fille, le fïls d'un fermier important de Condrieu (Rhône) a dû passer, en 1408, près de cinq mois en prison, avant que ne s'ouvre son procès $^{18}$. Et Jacquier de l'Arbresle doit affronter un interrogatoire serré de la part des officiers royaux à cause d'une servante d'auberge, dont on prouvera ensuite le mensonge et la coupable complicité avec des concurrents de Jacquier ${ }^{19}$.

\section{Éviter les «folles dénonciations »}

Pour pallier cet inconvénient, la justice a dressé deux garde-fous. Le premier consiste à fulminer des sanctions extrêmes contre les fausses accusations. Les femmes qui, effrayées par leur audace, songent à revenir sur leurs précédentes déclarations et qui se rétractent paraissent doublement condamnables, parce qu'elles ont mis une autre personne en péril de mort, et parce qu'elles ont manifesté leur irrévérence vis-à-vis des juges. On ne sait quel argument détermine le plus sûrement la peine de fustigation et de bannissement qui les atteint en général.

Mais la meilleure assurance contre le mensonge provient sans nul doute de l'enquête minutieuse sur la personnalité de la victime. qui est entreprise lors du dépôt de la plainte. Celle que mène Jacques Borestel. clerc de la mairie de Dijon, en février 1472. concerne une certaine Jehanne, femme de Jehan de Relange. Il s'agit de savoir «selle est bonne proude

16. ADCO, BII 360/13, 27 avril 1477, affaire Marguerite, fille de Perrenot Bargain.

17. ADCO, BII 360/01, pièce $n^{\circ} 7$, octobre 1421, affaire Gillote, épouse de Thévenin de Mons.».

18. Archives départements du Rhône, 10 G 2246, Condrieu, 1408, affaire Jeanne Genoi.

19. AD. Rhône, 10 G 603, f" 80, Lyon, 8 novenbre 1432. 
femme mariée ou se elle est temme ligiere, commune, secrete, pour et ala charge ou descharge des dessusnommez qui l'ont prinse, batue et ravie a force et violenté $\mathrm{e}^{20} \gg$. Les témoignages destinés à préciser la réputation de la victime n'occupent pas moins de vingt-cinq folios: quinze voisins et voisines apportent leur touche au portrait que l'on fera desormais de ladite Jehanne ${ }^{21}$.

\section{PROUVER LE VIOL}

Au-delà de cette phase de l'enquête, le sérieux de la dénonciation devra recevoir une démonstration rigoureuse. C'est pourquoi la première déposition recueillie est celle de la victime, à qui l'on demande de prouver le viol. La question demeure la même, à travers les siècles, dans ce type d'instruction : il s'agit de faire une nette différence entre acte consenti et forcé.

\section{L'usage de la force}

Le vocabulaire contribue déjà à replacer l'acte dans toute son horreur : les violeurs ont «pris à force», « ravi et forcée». "cogneu charnellement malgré elle et a forcé », « tenu et chevauché », «efforcé » leur victime.

Le récit des circonstances du viol vient étayer cette version. Jeanne Genoi raconte son infortune devant les officiers de Condrieu. Elle a rencontré Étienne Girerd dans un lieu isolé. alors qu'elle ramenait les boeufs du pré. Étienne l'a couchée à terre, lui a lié les pieds à un arbre avec de solides cordes et a pu ainsi accomplir son coupable dessein 22 .

Richote expose la manière dont son maître. Hugon Fèvre, l'a surprise dans son lit, «la print par les bras et les lui cuidoit mectre dessoubz le doz et fasoit la plusgrant force qu'il povoit de soy bouter entre ses jambes [...] et pour ce icelluy Hugon lui meist le genoul sur la poictrine ${ }^{23} »$. Gillote. l'épouse que trois hommes ont enlevée et violée, explique que «tandis que l'un la chevauchait, les autres deux la tenoient par les bras et les jambes et lui bouchoirent la boiche pour ce qu'elle crioit». Un témoin ajoute même qu'ils n'ont pu l'emmener ainsi que sous la menace, lui pointant «leur

20. ADCO, BII 360/12, 21 févricr 1475, affaire Jeanne de Relange.

21. Cf. GONTHIER. N., «Mala fama et honneste conversacion, les critères de la morale populaire d'après les sources judiciaires aux $\mathrm{xiv}^{\mathrm{e}}$ et $\mathrm{xv}^{\mathrm{e}}$ siècles $»$, dans «Ordre moral et délinquance de l'Antiquité au $x x^{e}$ siècle ", $2^{2}$ Colloque du CEH. Université dc Bourgugne, 6-7 octobrc 1993, Dijon, pp. 33-46.

22. AD. Rhône, $10 \mathrm{G} 2246, \Gamma^{\circ} 7$, Condrieu, 1408.

23. ADCO. BII $360 / 01$, pièce $\pi^{\circ} 9,12$ juin 1432, aftaire Richotte, fille de Perrenin Mercerot. 
espées contre son corps pour ce qu'elle crioit et qu'elle ne vouloit cheminer à leur plaisir $24 "$.

Les viols ou les tentatives de viols s'accompagnent en effet de violence. Celui qui ne peut aboutir à ses fins se prend à battre la femme rebelle. Nicole Blaise en a fait l'amère expérience, que son agresseur a battue et frappée de «plusieurs cops de poingt sur la teste, dont elle cracha le sang ${ }^{25} \gg$. Les coups servent tout autant pour obtenir l'abandon d'une farouche résistance. La servante Jehannote que se partagent plusieurs compagnons a essayé de se «revencher» mais son violeur «la frapa plusieurs cops du poing sur la bouche et un cop de pommeau de sespée sur son front dont elle est ung peu navrée ${ }^{26}$ ".

La contrainte reste un élément déterminant dans la définition du viol, à tel point qu'une prostituée peut prétendre avoir eté violée ${ }^{27}$. Comment comprendre autrement, la plainte déposée par l'écuyer Claux de Fromont. "fiancé» et souteneur de Jehannote de Briesville, qui déclare que sa mie a été ravie et forcée par trois ou quatre compagnons. La vertu de la dame semble bien mince puisqu'elle avoue avoir connu plusieurs amants «de son bon gré et pour gaingnier», sur ordre dudit écuyer, mais cela ne l'empêche pas d'ajouter que les compagnons en accusation «la prindrent a force et oultre son grey», aussi «se plaint elle comme efforcée ${ }^{28} »$.

L'usage de la force et de la violence qui rend le viol possible n'emporte pas seul la conviction des juges. Il faut que la victime ajoute à son récit des preuves d'une réelle résistance de sa part.

\section{Les cris}

Elle devra persuader l'officier instructeur qu'elle a crié, tenté de crier ou que ses cris n'ont pu être entendus en raison de l'éloignement. trois cas de figure que soulignent les dépositions diverses. La servante enlevée de force appelle désespérément ses maître et maîtresse : «Sire. Sire, Dame, Dame. l'on m'anmainne pour Dieu, venez vers moy!». Des témoins requis l'ont entendue crier «Hélas que feraige?», ou bien «Messires, je vous crie mercy, pour Dieu, laissez moy" ou encore "A la mort. a la mort, las où me menes-vous ? ${ }^{29} \%$. Ces cris sauvent parfois celle qui les profère. telle

24. ADCO, BII 360/01, pièce n" 7, octobre 1421 .

25. ADCO, BII 360/08, piece $n^{\circ} 29,3$ juin 1462.

26. ADCO, BII $360 / 01, n^{\circ} 8$, pièce $n^{\circ} 3,22$ avril 1431 .

27. Ce cas est soumis a diverses interprétations sclon les tribunaux: certaitss coutumiers conçoivent la possibilité d’une indemnilé pour la prostiluée ainsi agressée, la jurisprudence du Châtelet démontre au contraire unc assimilation de la prostituéc à n'importe qu'elle autre victime, en cas de viol. Sur ces points $f$. A. Porteau-Bitker, article citê, pp. 521-522.

28. ADCO. BII $360 \%$ (0) pièce $n^{\circ} 5,1^{\text {er }}$ aûut l $40 \%$.

29. ADCO, BII 360\%1, n" 8, piece n" 3, 22 avril 1431 . 
Richote qui «doubtant que (ledit Hugon) ne la deshonorast, se print à braire et a crier tresfort par plusieurs fois ${ }^{30} »$. Mais bien des agresseurs empêchent leur victime de s'exprimer en la bâillonnant. Quand on demande à Marguerite. âgée de quinze ans, pourquoi «elle ne cria pas à la première fois ni à la seconde» puisqu'elle a fait le récit de deux viols consécutifs, elle dit que son maître «luy tenoit la main sur la bouche, et ne peust oncques crier $^{31}$ ». Les mêmes explications reviennent dans plusieurs instructions. Mais des moyens plus persuasifs réussissent à étouffer les cris : la servante de François le Clerc qui a dû suivre ses ravisseurs dans toute la ville de Dijon. jusqu'au bordel, explique qu'elle «alait tousiours plorant et n'ozant dire mot pour ce qu'ilz luy disoient que se elle disoit mot. ilz luy copperoient la gorge $^{32}$ ». Ce genre d'argument n'est pas de ceux que l'on peut prendre à la légère car - on l'a vu - les violeurs se montrent capables des pires violences $^{33}$; aussi réduit-il au silence également la jeune Catherine à laquelle son agresseur promit qu'il la tuerait de son couteau si elle criait $^{34}$. Intermittents ou étouffés, les cris de la victime restent une preuve majeure de son innocence.

Par contraste, l'enquête menée dans l'affaire du rapt et du viol présumés d'une certaine Marguerite, servante à Dijon, recueille une infinité de dépositions attestant que les voisins immédiats n'ont «oy aucun cry ne clamour». Chaque question de l'officier cherche alors à vérifier si le témoin était bien présent le jour du crime et à quelle distance exacte du lieu où il s'est produit se trouve sa maison. Jehannote, par exemple, précise qu'elle était au cellier de son hôtel avec d'autres, ce jour où elle aurait dû entendre quelque chose: Henriot le Fonassey, "voisin à deux maisons», confirme que « la sepmaine que l'on dit que ladite Marguerite fut prinse et emmenée, il fut continuellement senz lui departir en sond hostel et en la rue, et par jour et par nuit »; et ainsi de suite. pour au moins vingt-trois des vingt-huit témoins produits, les derniers avouant qu'ils n'ont glané que des rumeurs car ils habitent trop loin de la demeure de la victime ${ }^{35}$.

La charte des franchises de Trévoux, près de Lyon. comme d'autres semblabies, dans la région, prévoient pareille contradiction entre l'accusation et les témoignages et conclut que «si puella vel aliqua mulier dicit

30. ADCO, BII $360 / 01$, piece $1^{\circ} 9,12$ juin 1432 .

31. ADCO, BII 360/04, n'1, pièce n" 358, 22 mai 1449.

32. ADCO, BII 360/11, 10 janvier 1467.

33. Notre disumentation ne mentionne cependant aucun meurtre de la part des violeurs.

34. ADCO, BII 360/0I, pièce n 16, 12 mars 1418.

35. ADCO, BII 360/1. pièce n” 3, 11 lévrier 1404, alfaire Marguerite, fille de lécu Colas Damence. 
sibi violentiam illatam fuisse ab aliquo, in tali loco ubi potuerit clamare et audiri ab aliquibus, si non clamaverit non debet esse credi $i^{36}$.

Ne pas être entendue, voila bien la pire chose, car non seulement aucun secours n'interrompra le viol, mais encore une preuve essentielle de celuici vient à manquer. C'est cette argumentation que, malgré son défaut manifeste de perspicacité. la jeune Jacotte avance, après être tombée dans un piège grossier. tendu par une maquerelle. «Elle se prit a crier [dit-elle, mais elle] ne peust estre oyée parce que elle estoit tout derriere l'ostel [de Vergy $]^{37}$ ".

Se « revencher»

Les officiers instructeurs comme les juges apprécient également de pouvoir évaluer le degré de combativité de la victime. la passivité en ce domaine figurant comme une marque de consentement. Pour cela, ils suscitent, à force de questions adroites, un exposé précis des divers obstacles mis à la satisfaction du violeur. La veuve de Martin Porte. de Chasselay (Rhône), qui a subi l'attaque de deux compagnons, insiste sur le fait qu'elle avait clos sa porte d'une solide barre de fer avant d'aller se coucher. La barre de fer a cédé, de même qu’ont été arrachés par les furieux les piquets de la clôture du jardin et que rien ne résista dans la chambre, quand ils fouillèrent pour trouver la femme convoitée, comme on débusquerait un

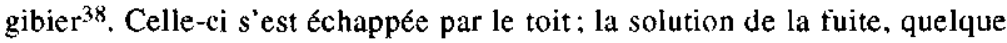
danger qu'elle représente, revient souvent dans les dépositions. Une tenancière d'hôtel. à Dijon, énumère les diverses dégradations que trois ou quatre hommes ont causé à sa demeure "pour la prendre et l'avoir afín d'en faire leurs plaisirs". Ayant frappé à l'huis sans qu'on leur ouvrît, ils se sont employés à rompre la serrure d'une dague : n'y parvenant pas, ils ont utilisé une grosse pièce de bois comme boutoir et en «rompirent les parois de la maison ", puis ils forcèrent encore la porte de sa chambre ! La propriétaire, entre-temps, telle la veuve Porte à Chasselay, avait quitté les lieux "par un pertuis de ladite maison», sauté dans le jardin đu voisin, «dont-elle a la jambe toute escourché». Mieux vaut une jambe écorchée à la flétrissure du viol - semble-t-elle souligner - pour inculper davantage ceux qu'elle a dénoncés ${ }^{39}$.

36. VALENTIN-SMITH, M.M, et GUIGUE M.C., (1854-1885), Bibliotheca Dumbensis ou Recueil de chartes, titres et documents pour servir à thistoire des Dombes, I. I, Trévoux, p. 44, arI. XLVI.

37. ADCO. BIl 360/8. 10 août 1468, affaire Jacottc, fille de feu Alexandre de Chastelvilain.

38. AD. Rhònc, 11 G 693, 1" 157, Chazay d'Azergue, 1412.

39. ADCO, BII 360)/1, 27-28 aût 1422. 
Quand la fuite est impossible, il reste à la femme à se battre contre le violeur. Nicole Blaise affirme s'être «deffendu ainsi qu'elle pouvoit» en croisant « les jambes qu'elles avaient mises l'une sur l'autre» et en mordant son agresseur, très fort «en ung doit de la main qu'il lui tenait sur la bouche $\mathrm{e}^{40}$ ». Perrotte Bidon raconte combien «elle regippoit des piedz et se deffendoit comme elle pouvoit ${ }^{41} »$. Cette formule revient de façon répétitive. Dans son imprécision, elle contient toute l'impuissance tragique de ces filles ou de ces femmes que la violence finit par soumettre.

\section{Dire le viol}

Quand elles ont présenté les circonstances du viol et la résistance qu'elles lui ont opposée, les victimes de ce crime doivent encore franchir une épreuve supplémentaire qui consiste à décrire l'acte afïn que la cour puisse évaluer le degré de gravité qu'il a atteint.

«Dire le viol" reste encore aujourd'hui - si l'on en croit les statistiques criminelles contemporaines - l'obstacle majeur à la plainte formée. La même répulsion se manifeste au $x v^{e}$ siècle. Jeanne de Relange est soupçonnée d'avoir commis une «folle dénonciation» car elle a varié dans ses accusations contre les compagnons de guerre picards qui l'avaient violentée. Quand on lui demande de s"expliquer sur ce point, elle avoue que «tant de fois a esté interroguée et par tant de gens qui luy ont demandé se lesd. picard $z$ avoient point semé leurs semences sur elle comme dit est, qu'elle en a esté tant honteuse et tant atainnée et a eu si grant horreur des ordes paroles et de reiterer la maniere a ceulx et celles qui luy en ont parlé et demandé, qu'elle a dit et repondu aulcunes fois [...] que l'on la laissast en paix et que lesd. picardz ne luy avoient riens fait $^{42}$ ».

On a vu plus haut combien la publication du forfait pouvait tarder en raison de la honte ressentie par la victime. Or la rapidité avec laquelle elle vient le dénoncer est reçue comme une preuve de son innocence, comme l'assurance qu'elle ne fut ni consentante, ni complice. C'est un avis «de bon sens», partagé par les juges et l'opinion publique, ainsi que le confirment certaines lettres de rémission - documentation complémentaire aux instructions qui vise moins cependant à présenter l'exacte réalité. qu'à transmettre des «images conformistes » du criminel ou de sa victime. Dans sa demande de rémission à la duchesse de Bourgogne, un violeur qui plaide non coupable, prétend prouver qu'il n'y a pas eu viol puisque sa victime «ne scest mye venu doloir incontinant deschevelée ne autrement ainsi que en tel cas appartient mais a atandu jusques le lendemain ${ }^{43}$ ». Telle doit donc

40. ADCO. BII $360 / 8$, pièce n" 29,3 juin 1462 .

41. ADCO, BII 360/03, pièce $n^{\circ} 242,6$ juillet 1444.

42. ADCO, BII 360/12, 21 lévrier 1472.

43. ADCO, BII 360/01, 9 janvier 1426. 
apparaître la victime du viol : sans coiffe, «en cheveux», selon l'expression dont on usera au XIX siècle. c'est-à-dire dépourvue de ce qui établit distinction et honneur ${ }^{44}$, et dans cet état de total anéantissement. elle doit illico confesser les tourments qu'elle vient de subir. Les comportements des femmes violées s'écartent souvent de ce modèle.

\section{Des mots pour le dire}

Les officiers chargés de l'instruction obtiennent cependant une description très réaliste du viol. Le vocabulaire employé. le scénario dévoilé. se veulent d'une exactitude clinique. sans ambiguïtés.

Écoutons Jehannote : «led. seigneur la print et la coucha malgré elle sur ung lit qui estoit en icelle chambre et incontinent la descouvra et desvala ses petits dras et se bouta entre ses jambes [...] il luy appointa son membre contre sa nature et se parforça de le bouter dedans ${ }^{45}$ ». Plus précis encore est le récit de Catherine Malonne, dix ans: «et comme il l'eusse couchée, desvala ses braies, la descouvrit, se boutit entre ses jambes et lui bouta son vit au con ${ }^{46} »$. Dans ce genre de rapport apparaît très nettement ce que l'on nommerait aujourd'hui le niveau d'«information sexuelle» de chacune des victimes. On opposera aisément la personne de Richote, dont les seize ans sont déjà prévenus contre les dangers qui guettent les servantes, et une autre domestique, presque du même âge. Marguerite Guillème, qui manifeste dans sa déposition une naïveté et une ignorance extrêmes, proches de la stupidité. Richote en effet n'hésite guère à décrire la nudité de son maître quand il vint dans son lit et l'érection de son sexe : «ledit Hugon avoit mis nulx petiz draps mais avoit son membre bien roide qui lui bouta contre le cousté ainsi comme il cuidoit bouter ses jambes entre les siennes ${ }^{47} \gg$, tandis que le récit de Marguerite démontre qu'elle n'a pas conçu le danger ni compris ce qui lui arrivait. Elle semble n'avoir éprouvé qu'étonnement quand Humbert le Ravet. son mâ̂tre, l'emporta, nue, du lit où elle dormait jusque dans le sien. Elle décrit l'acte sexuel comme une gestuelle incompréhensible. Son vocabulaire est celui d'un enfant au contact d'un comportement d'adulte. "Il lui ouvrist de l'aultre main les jambes et se mist de son long sur elle et prinst son membre lequel il lui bouta en sa nature a grant force et peine. Et apres se remua sur elle bien longuement et lui semble qu'il y fus bien une demy heure». Elle ne

44. Dans le rapt de Jehannote, servante de Jean Morel, pour laisser croire qu'il garda respect vis-à-vis de ladite femme, un des inculpés ćvoque l'incident suivant : «en Ia menam, les chapperons el coiffe de la dicte Jehannote li cheurent d̀ terre, lequel qui parle les releva et les rebailla à lad. Jehannote». ADCO, BII 360/01, n'8, pidce $n^{\circ} 3$, 22 avril 1431.

45. ADCO, BII 360/01, $\mathrm{n}^{\circ} 8$, pièce $\mathrm{n}^{\circ} 3,22$ avril 1431.

46. ADCO, BII 360/0l, piece n"16, 12 mars 1418 .

47. ADCO, BII 360/ $/$, picce n" 9, 12 juin 1432. 
reconnaît pas davantage le sperme qui «la lassa toute moillée dedans et dehors sa nature» ni ne s'explique le sang qu'elle vit le lendemain sur les draps. Elle perçoit si peu les graves conséquences de sa passivité qu'elle se laisse surprendre encore deux fois, selon le même scénario, par Humbert ${ }^{48}$. Alors que Richote parle d'éviter le déshonneur, Marguerite n'a pas même conscience de sa propre défloration ni ne connaît. sans doute. le mot de «viol». À quinze ans, elle semble n'avoir pas plus de notion du sexe que la fillette de quatre ans, victime d'une tentative de viol. dont on nous dit qu'après avoir été mouillée du sperme du violeur, elle s'en alla se plaindre à sa mère que l'homme «l'avoit compissiéc49».

Naïves ou non. les déclarations des victimes sont donc, on le constate, extrêmement concrètes. L'enquête réclame encore un compte exact des accouplements subis et les victimes donnent sur ce point les informations souhaitées. Pernotte, servante de François le Clerc, violée une première fois par l'un de ses ravisseurs, dut passer la nuit au bordel avec un autre d'entre eux «qui eust encoires compaignie a elle charnellement quatre ou cinq fois ${ }^{50}$ ». Quant à Jehannote, victime du même type de rapt, elle est déflorée par l'un, puis livrée à deux autres compagnons qui « la cogneurent charnellement chascun deux fois ${ }^{51}$ ». Mais rien n'égale le langage de Jacotte. dix-sept ans, pauvre fille employée au service des pauvres, à l'hôpital de la Magdeleine à Dijon, lorsqu'elle avoue que le violeur : «eust compaignie charnelle avec elle par deux fois, à deux montées et à deux descendures ${ }^{52}$ ».

\section{Des preuves supplémentaires et aggravantes}

Dans le récit même. ou par des questions «annexes». les officiers trouvent encore des preuves supplémentaires du viol.

La plus fréquente de leurs interrogations, concerne la sensation ressentie par la victime : «douleur ou plaisir»? Le terme utilisé pour décrire de l'homme la jouissance satisfaite reste «quand il eust ainsi fait son plaisir d'elle» mais à la femme on demande : «se en ce faisant elle print aucun plaisir ${ }^{53}$ ». Cette question est posé de nouveau aux coupables du viol, lorsqu'on écoute leur version des faits, et l'on gage bien sûr qu'ils ne répondent pas par la négative! Simon le Verpillet. qui a détloré la jeune Catherine, âgée de dix ans, se voit requis de dire «se en faisant l'auvre dessus dit. ladite Katherine se plaignoit point». Il prétend que non ${ }^{54}$. À Jacotte, qui a subi deux assauts successifs, on demande « $s$ 'il luy fit pas de

48. ADCO, BII 360/04, $n^{\circ} 1$, pièce $n^{\circ} 358$.

49. ADCO, BII 360/04, piece $\mathrm{n}^{\circ} 376,23$ septembre 1449.

50. ADCO, BII 360/11, 10 janvier 1467.

51. ADCO, BIl $360 / 01, \mathrm{n}^{\circ} 8$, pièce $\mathrm{n}^{\circ} 3,22$ avril 1431.

52. ADCO, BII 360/8, 10 a)ût 1468.

53. ADCO, BII 360/01, $\mathrm{n}^{\circ} 8$, pièce $\mathrm{n}^{\circ} 3,22$ avril 1431.

54. ADCO, BI! 360/01, piece $n^{\circ 1} 16.12$ mars 1418. 
mal à la première fois ». Elle répond que «oy ${ }^{55}$ ». D'elle-même. la victime, lors de sa déposition. a parfois évoqué la douleur de la défloration ou celle d'un rapport exigé avec trop de violence. Jehannote expose que «pour ce [...] qui lui faisoit grant mal, se prist à crier en lui criant marci ${ }^{56} »$. Catherine Malonne confirme encore : «Il luy fist moutl mal et tellement qu'il la feist saignée tresfort ${ }^{57}{ }$. La jeune Perrotte Bidon, enfin, a eu la sensation qu'on lui crevait le ventre et ajoute que le violeur : «luy fit tant de mal et de peine $^{58} \gg$.

Après s'être enquis ainsi des signes d'une défloration possible, les officiers instructeurs posent une seconde question, liée logiquement à la première: la victime «a-t-elle eu jamais compagnie charnelle à un homme»" À quoi. le plus souvent. il est répondu que non et que la fille était pucelle avant l'attentat.

En s'exerçant sur une vierge, le viol gagne une gravité supplémentaire. Les tribunaux de l'église de Lyon, responsables de la justice ordinaire dans la ville et ses campagnes, insistent à plusieurs reprises sur ces considérations qui incitent les juges à des sanctions exemplaires : «virginitas corrupta est enormis crimen ${ }^{59}$ ". La justice laïque n'est pas moins sensible aux conséquences sociales de ce crime, puisqu'il retire à la jeune femme le seul avantage qui pouvait, en dépit de son peu de fortune, en faire une épouse acceptable. Cours épiscopales, royales, échevinales s'entendent sur ce point et s'inspirent peut-être des écrits de Thomas d'Aquin à propos de la défloration d'une vierge, acte que le saint désignait avec dégoût du terme de «stupre ${ }^{60} »$.

La pucelle violée, éventuellement partagée entre des complices, est désormais perdue de réputation, assimilée aux ribaudes. La mère de l'une d'elles clame à tout le voisinage que Jehan Le Dienet, religieux de SaintJean de Jérusalem «li (a) vergondée sa dite fille ${ }^{61} »$. Aux accusations que cette mère éplorée fait entendre devant la cour échevinale de Dijon s'associent les saurs et les frères de la victime. C'est en effet l'honneur de toute une famille qui est compromis, comme le prouve assez le comportement du père de Jeanne Genoi. «efforcée » par Étienne Girerd à Condrieur. Refusant les indemnisations que lui offre le père du violeur, il clame que «lui, sa fille

55. ADCO, BII 360/8, 10 août 1468.

56. ADCO, BII $360 / 01, n^{\circ} 8$, pic̀ce $n^{\circ} 3$.

57. ADCO, BII $360 / 01$, pièce $\mathrm{n}^{\circ} 16$.

58. ADCO, BII 360/03, $\mathrm{n}^{\circ} 242,6$ juillet 1444.

59. AD. Rhône, $10 \mathrm{G} 2246, \mathrm{f}^{\circ} 7$, Condrieu, 10 décembre 1408.

60. Dictionntire de Théologie catholique, I. VI, article «Fomication», coll. 600611 .

61. ADCO, BII 360/1, pièce $\mathrm{n}^{\circ}$ 3, I I février 1404 . 
et ses autres filles et parents sont diffamés et injuriés à tel point en raison de cette infamie et de cette faute que Jeanne ne pourra jamais se marier ${ }^{62}$ ».

Alors que l'atteinte à la réputation paraît un tel facteur aggravant du crime. la justice se soucie également du dommage physique subi. L'apprécier constitue certes une ultime preuve du viol mais permettra surtout de moduler l'attitude des juges à l'égard du coupable. Des spécialistes sont commises à cette tâche : il s'agit de «femmes appelées matrones qui ont accoustumé de recevoir des femmes les enfans». Elles pratiquent une véritable médecine légale, puisqu'elles agissent après avoir prêté «serrement sur sains evangilles de Dieu qu'elles rapporteroient verité de ce qu'elles en trouveroient». L'inspection des matrones que l'on nomme «reverchiement " dans la langue d'oïl en usage à Dijon, donne lieu à des rapports détaillés et précis. On exige de ceux-ci qu'ils déterminent d'une part si la victime a été déflorée et "cogneue domme [d'homme] charnellement et nouvellement», d'autre part l'état physique dans lequel l'efforcement l'a laissée. C'est ainsi qu'après avoir examiné Jeannote, les matrones concluent qu'elle «avoit esté et estoit frachement cogneue domme par grant violence a force et quelles avoient veu et cogneue femme qui avoit eu trois enfans qui n'estoit pas si corrompue domme ${ }^{63} \gg$. Pour Perrote Bidon, elles donnent un diagnostic moins inquiétant mais tout aussi scrupuleux : «l'en y avoit habité et y estoit entré mais non pas si avant qu'il luy rompist la peaulote mais il s'en aida tellement qu'il y laissa sa semence ${ }^{64} »$. Cependant. les matrones ne sont pas les seules capables d'effectuer ce bilan clinique. Toutes les femmes mariées savent le dresser et le cours de l'enquête peut en être faussé. Nous voyons la femme Thévenin, pour étouffer l'affaire qui a eu pour cadre son hôtel et mis en cause l'un de ses valets, pratiquer elle-même, avec deux de ses servantes, le «reverchiement» de l'enfant qui se plaint d'avoir mal. Ayant reconnu le viol, elle fait baigner «par III fois» la fillette. Quand n'intervient pas la volonté d'effacer les preuves, c'est parfois en apportant les premiers soins à l'infortunée victime que le zèle des femmes contrarie le travail des matrones. Pour Perrotte Bidon, la matrone signale qu'elle «ny vit point de sang» mais qu'elle «ny peust avoir bien simplement la congnoissance pour plus avant en declairer » car la fillette ne peut supporter qu'on lui touche «le lieu de la nature " tant son agresseur l'a heurtée et tant «les femmes l'avoient ja torchiée et nettoyée ${ }^{65}$ »

La compétence des sages-femmes doit s'appliquer en outre à soigner la victime. Les souffrances de celle-ci, et même la maladie que le viol a

62. AD. Rhône, $10 \mathrm{G} \mathrm{2246,} \mathrm{10 \%} \mathrm{7,} \mathrm{Condricu,} 10$ décembre 1408.

63. ADCO, BII 360/01, n" 8 , pièce $n^{\circ} 3,22$ avril 1431.

64. ADCO, BIf 360/()3, $n^{62} 242$. 6 juillet 1444.

65. Hidem. 
provoquée, figurent comme des preuves supplémentaires du crime, collectées par les enquêteurs. "A l'occasion des violences et forces dessusd. n'a-t-elle point esté malade?» La question revient de façon systématique parmi celles qui sont destinées à compléter certains aspects de la déposition. La femme violentée répond « oy» et ajoute, en général, qu'elle souffre encore de ce mal. Elle reste "très fort malade et cassée en sa nature», comme se lamente Catherine Malonne. Cependant. Jehanne de Relange va plus loin dans le compte rendu des conséquences malheureuses du viol quand elle avoue être "malade au lieu secret de nature [...] quel maladie elle a, dit quelle est honteuse de le dire et seroit afere à femmes de la visiter pour le dire» mais il «est vray qu'elle est si malade qu'elle brule toute dedans le corps [...] dont [d'où] lui procède lad. maladie? elle ne scet se elle ne procede et vient des oppressions, talures, folures et travailz qui luy furent faictes rudement par ceulx qui la coucherent en la naige pour ce qu'elle ne leur vouloit souffrir fere leur plaisir dampnable, ou que l'un deulx en fust malade ${ }^{66}$ ». Pour soigner ces maux les matrones fabriquent des emplâtres avec des herbes.

\section{LES VIOLEURS}

Ces «preuves médicales» marquent en principe la clôture de l'interrogatoire de la victime. Mais elles sont utilisées avec habileté pour confondre les auteurs du crime. C'est d'eux désormais que se préoccupe l'enquête. Notons toutefois que, dès la réception de la plainte. ils ont été incarcérés et qu'ils ne bénéficient pas du système de la libération sous caution qui s'applique en d'autres affaires criminelles. Ils ont donc attendu, en prison, que l'enquête s'achève et établisse la certitude du viol ou bien déboute la plaignante. Ce séjour dans les cachots peut s'avérer fort long - quelque cinq mois dans l'affaire Jeanne Genoi. pour Étienne Girerd de Condrieu. Du fond de leur cellule. les prévenus osent parfois lancer une demande de rémission, tel ce Jehannin Boiseau, natif de Normandie mais demeurant a Dijon, âgé de vingt-cinq ans. Incarcéré depuis le mois de décembre 1426, il lance, le 9 janvier 1427, une supplique à la duchesse de Bourgogne, Isabelle de Portugal, de ce que Monseigueur le mayeur «l'a fait prendre et mectre es prisons dud. lieu [...] esquelles il est en grant misere et est taillé d'y demourer longuement» à moins que la grâce ducale ne le sauve ${ }^{67}$.

\section{Leur portrait par les victimes}

Avant que les enquêteurs ne leur donnent l'occasion de s'exprimer, en les interrogeant, les violeurs nous apparaissent à travers les dépositions de

66. ADCO, BII 360/12, 1477.

67. ADCO, BIJ 360/01, 9 janvier 1426 (Ancien Style). 
leurs victimes. Les portraits qu'elles en donnent ne sont guère à leur avantage.

On y voit en effet des maîtres d'âge mûr qui perdent toute notion de la décence devant leur jeune servante, venant en chemise jusqu’à leur couche. détournant leur autorité a des fíns malsaines, usant de la menace et terrorisant des innocentes, se faisant suborneurs à l'encontre de celles qu'ils devraient protéger comme un père. Quel hiatus ne ressent-on pas dans la personnalité de Hugon Fevre? Ce notaire public cache en réalité une nature travaillée par la luxure, réclamant à son voisin qu'il lui fournisse une ribaude pour une nuit, puis se laissant convaincre qu'il aurait meilleur compte à débaucher sa servante. Le crime d'Hugon commence là, dans la rue. devant son hôtel, quand il partage des discours lascifs avec ses compagnons, à propos d'une jeune fille de seize ans, dont la conduite ne prête à aucune infamie, quand il lance ensuite à celle-ci des propositions malhonnêtes, en public. «Se tu veulx encoires couchié avec moy, je te donray ung bon chapperon, voire, par Dieu, la robe avec ${ }^{68} »$. Ces paroles n'ont qu'un but, diffamer la future victime, afin que sa plainte ne soit pas recevable. Cette tactique honteuse, d'autres l'adoptent fréquemment. Jacques Rossiaud a montré comment des coups à la porte d'une femme en pleine nuit, des quolibets lancés à son adresse dans la rue, des rumeurs mensongères répandues, préparaient souvent un viol collectif, car la mauvaise réputation acquise ainsi par la victime découragerait les voisins d'intervenir pour sa défense ${ }^{69}$. La tentative de viol dont se rend coupable ledit Hugon a donc été préméditée; elle révèle une obsession perverse qui lui vaut les insultes de sa voisine, une femme en âge de lui dire ses quatre vérités. Les épithètes de «sanglant conchies», de «putier», de «traicte», d"«homme de neant» sanctionnent en lui le luxurieux et l'hypocrite.

Le personnage d'Humbert le Ravet n'est guère plus reluisant qui profite des absences de son épouse pour commettre l'adultère avec sa servante et que la naïve description de celle-ci présente «soufflant et suant» tandis qu'il peine à satisfaire ses désirs coupables ${ }^{70}$. Ces bourgeois respectés, qui pratiquent ainsi le "stupre" dans le secret de leurs hôtels, manifestent le plus grand mépris pour leurs victimes. Aucun regret de leur part, mais une colère quasi homicide quand leurs désirs sont contrariés.

C'est encore ce mépris, doublé d'une certaine cruauté, que l'on rencontre chez une autre catégorie de violeurs : les compagnons de guerre ou les bandes armées dont les rapts et les viols résultent d'une action concertée. conçue comme un jeu, une chasse ou une expédition guerrière. La victime. traitée comme un butin que l'on partage, dont on use sans réserve.

68. ADCO, BII $360 / 01$, pièce $n^{\circ} 9,12$ juin 1432 .

69. ROSSIAUD, J., La Prostitution médiévale.

70. ADCO, BII $360 / 04, n^{\circ} 1$, pièce $n^{\circ} 358,22$ mai 1449. 
ne peut compter sur aucune pitié; au contraire il semble que ses pleurs excitent des tendances «sadiques» parmi ces criminels.

Un dernier type $d$ 'agresseurs se recrute principalement chez des valets ou des compagnons dont l'acte paraît moins prémédité que dans les deux précédents cas. Au contact permanent des servantes ou des filles de ferme dans les travaux quotidiens. ils cèdent à des impulsions subites, irrépressibles. Le décor de leurs violences, c'est la grange. l'étable, le cellier, la vigne; le viol a ce caractère furtif d'une jouissance interdite, dérobée à la foule importune des autres domestiques. Il n'est pas question là de fille tenue toute une nuit, comme le font les compagnons armés, ni d'assauts répétés. comme se le permettent les bourgeois.

\section{Leur version des faits}

Mais quel que soit le «profil» des violeurs, ils tiennent à peu près tous le même langage quand on les interroge sur leurs agissements. Leurs dépositions tendent d'abord à présenter la femme violée comme une partenaire consentante et non une victime. L'argument reste la défense des violeurs d'aujourd'hui. Il figure dans l'instruction du procès pour viol concernant Jehannote de Vauls, servante de Jehan Morel: un des prévenus, Audrieu Viart. vingt-deux ans, fait de celle-ci le portrait d'une ribaude qui ne cria pas lorsque le chef de la bande l'emmena dans la chambre mais «monta les degrez de son bon gré et volenté» et qui exigea même d'y rester avec deux hommes, alors qu'ils lui avaient donné "congé», allant jusqu’à refaire le lit. après quoi elle «se coucha en icelli et lui qui parle et ledit Audrieu du Vernoy se couchèrent avec elle et la cognut lui qui parle, six fois, et led. Audrieu ne scet quante fois ». Se vante-t-il, ou veut-il insister sur l'insatiable goût pour la débauche qui caractériserait cette femme? Elle n'a reconnu, pour sa part, que deux accouplements de chacun. Il dit encore que Jehannote ne «crioit point mais les en prioit». Pour mieux asseoir le personnage de la fille publique. il ajoute qu'il lui demanda «selle avoit esté autrefois chevauchée. laquelle lui repondit que le verlet dud. Morel l'avoit despucellée passé avoit ung an ". Cette version avantageuse, qui ruine l'inculpation pour rapt, pour viol et pour défloration tout à la fois, est réitérée par l'autre prévenu Guillin Moisson. dix-huit ans ${ }^{71}$.

De telles putains ne sauraient être crues en jugement. C'est ce que glisse Jehannin Boiseau dans l'argumentation de sa demande de rémis$\operatorname{sion}^{72}$. Elles cultivent en effet le mensonge comme la luxure. Aussi la dénonciation de Guiote à l'encontre de Jehannin ne s'explique-t-elle que par la haine qu'elle a pour lui. car jusque là «elle a bien aimé led. suppliant et ...plusieurs autres bons compaignons qui d'elle ont fait leurs bons plaisirs

71. ADCO, BII $360 / 01, n^{\circ} 8$, pièce $n^{\circ} 3,22$ avril 1431.

72. ADCO, BII 360/01, non colć. 
par cople charnelle dont elle ne s'est mye plainte». Habilement, Jehannin renverse les accusations : Guiote aurait menti à la cour, commis une «folle dénonciation» par désir de vengeance. mené une vie de prostituée. Avec de pareilles ribaudes, plus de scrupules à conserver : on se vante de les avoir «connues charnellement», on emploie même un vocabulaire beaucoup plus avilissant pour exprimer ces rapports de domination masculine. Sollicités pour déterminer si la nommée Marguerite fut réellement ravie et déshonorée par quelques religieux de Saint-Jean-de-Jérusalem. certains témoins ont entendu l'un des religieux se féliciter en ces termes: «Par Dieu nous l'avons chevauchié et chevaucherons ${ }^{73}$ ". La volonté de dégrader la femme dont ils ont tiré leur plaisir reste un point commun de tous les violeurs agissant en compagnie. Ainsi se conduit le chef de la bande qui a enlevé la jeune Jehannote, puisqu'il jette sa proie à ses amis en leur disant qu'il leur amène «une beste».

Un autre moyen d'humilier la victime du viol consiste à lui proposer un dédommagement pécuniaire, après avoir joui d'elle, comme on rétribuerait une prostituée. L'un de ses trois agresseurs offre à Gillote, l'épouse de Thévenin de Mons, la somme de douze blancs, ce qui, de plus, représente une somme ridicule (un blanc égale cinq derniers, douze blancs, soixante deniers, c'est-à-dire cinq sous). Et quand elle les refuse en répétant ses menaces de les faire pendre, ils choisissent une ultime vexation: «Faut y tant parler de ceste ribaude, bailons lui d'une paule [une gifle] ou cu [au cul] car c'est son paiement », et de la frapper sur les fesses comme pour insulter encore ce corps qu'ils ont dénudé et meurtri ${ }^{74}$.

\section{Le traitement des violeurs en justice}

À la lumière de ces versions fort contradictoires d'un même événement, les officiers chargés de l'instruction rapprochent les preuves, les soupèsent et avec patience et intelligence obligent le violeur à revenir sur certaines de ses affirmations. La démarche apparaît très nettement dans le cas de Symonnet le Verpillet, convaincu de viol sur la petite Catherine Malonne.

Sa première déclaration. Le 12 mars 1418, présentait le viol comme une réponse à une attitude provocante de la fillette : elle l'aurait rejoint dans la grange où il coupait du bois ; lui-même aurait satisfait au besoin de se soulager car «son membre li tendait». Mais il ajoutait alors «qu'il n'y peust entrer parce qu'elle estoit trop jeusne». Aussi niait-il «connaître charnellement» ladite Catherine. Deux jours plus tard, le maire et les instructeurs se transportent de nouveau dans les prisons pour un second interrogatoire. Ils obtiennent une confession plus complète. Sans avouer 
davantage la pénétration, Symonnet reconnaît que son rapport avec l'enfant a provoqué une éjaculation : «il espancha et laissa courir de la semence de son corps entre lesdites jambes". Le 16 mars, nouvelle visite au prisonnier. Mais cette fois, la cour est en possession du rapport des matrones qui a confirmé la pénétration et la défloration. Symonnet abat sa garde ; ce qu'il niait quatre jours plus tôt, il l'avoue désormais, ainsi que les plaintes de Catherine et la violence qu'il lui imposa. Le 22 mars il réitère ces aveux. tout en ajoutant qu'il n'avait jamais eu «compaignie à lad. Katherine autrefois".

Pour chaque interrogatoire, le greffier prend soin de noter que les aveux ont été obtenus«de son plain gré et sanz. contraincte». Exceptionnellement, on n'a donc pas "géhenné » le prévenu, alors que la torture est couramment utilisée dans la procédure extraordinaire qui s'applique aux cas de la haute justice criminelle, tels que le viol. Sans doute a-t-on estimé que Symonnet était un de ces violeurs plus «pathologiques» que criminels.

La manière dont l'instruction recherche la cause de l'acte condamnable illustre tout à fait la qualité de la justice arbitraire — celle où le libre arbitre du juge peut moduler les peines en fonction des motivations du criminel ou du danger qu'il représente pour la société. Une autre affaire nous le prouve, qui relate une tentative de viol sur une enfant de quatre ans. Le coupable a quarante-trois ans. Il se nomme Jacquet Changeon, est valet chez un barbier à Dijon. Lorsqu'on lui demande de décrire les circonstances de son acte et d'en donner les raisons, il ne trouve qu'une explication : «Il fut tempté de l'ennemy comme il croit.» Tout s'est passé rapidement, en effet, comme dans un accès de folie perverse, jusqu'à l'éjaculation précoce qui a évité le viol. Plus intéressantes que le récit qu'il livre, dont le caractère sordide reste très vif, les questions qu'on lui pose vont essayer de cerner en lui la part de volonté maligne et la part «pathologique».

Pourquoi a-t-il fait choix de cette fillette si jeune? On tente ici de savoir s'il a un goût pervers pour les petits enfants. Il a obéi, déclare-t-il, à une impérieuse nécessité : «il estoit eschauffé et sa verge ly tendit». La question suivante essaie d'évaluer en lui les instincts du violeur : «Se il eust pu entrer en elle avant que nature feust partie de luy, il y fust entré ?» $\grave{A}$ une telle interrogation d' «histoire-fiction», il ne sait que répondre! Il invoque l'impossibilitế anatomique : «elle estoit trop petite et jeusne».

Devant ce cas aberrant, les officiers soupçonnent une frustration sexuelle. un déséquilibre qui dévoie les désirs de cet homme de leurs objets naturels et normaux. Cette intuition apparaît dans la dernière question consignée par le greffier: «eus-til oncques compaignie a quelconque femme commune ou autre? ? Lorsqu'ils entendent la dénégation de Changeon, leur opinion est faite. Il y a, dans ce viol, plus de dérèglement mental 
que d'intention consciente, mais cette constatation n'augure nullement la mansuétude des juges.

\section{Le sort des violeurs}

Les liasses ou les registres gardant mémoire de si longues et si minutieuses instructions mentionnent parfois les sentences publiées à l'issue du procès. On dispose, par ailleurs, de registres de sentences qui, sans reprendre le détail des chefs d'inculpations, en donnent un aperçu suffisant. À l'appui de ces sources, on ne peut que remarquer l'extrême sévérité des sanctions judiciaires. Quand le procès pour viol a été ouvert, il aboutit soit à une amende importante qui se chiffre en plusieurs livres ${ }^{75}$, soit à un bannissement, en général perpétuel, après quelques épisodes humiliants ou douloureux, telles l'exposition au pilori, la fustigation, la marque au fer ou la mutilation, soit enfin à une sentence de mort. C'est la décision prise par la cour échevinale de Dijon à l'encontre de Symonnet le Verpillet, condamné «a estre mors pendu et souffrir mort au darrenier supplice pour led. cas». La rigueur de la justice varie sans doute d'un tribunal à l'autre et selon les époques. Mais le viol reste un crime gravissime qui mobilise, on l'a vu, l'énergie des hommes de justice. Quelques coupables cependant réussissent à se soustraire aux pénalités, soit qu'ils bénéficient de l'appui de leur milieu $^{76}$ (Église, lignage noble, famille bourgeoise influente), soit qu'ils obtiennent un arrangement avec leur victime. La plupart de ces compromis échappent à notre investigation car ils font partie de ce que l'on nommera l'«infrajudiciaire» et ont été conclus oralement. Nous en gardons cependant l'écho dans certains documents, Jorsque les tribunaux officiels doivent trancher après l'échec d'un compromis, ou s'ils interviennent afin de conférer aux contrats «amiables» une valeur juridique plus ferme. C'est par ce biais que l'on connaît les tractations passées entre le père d'Étienne Girerd et celui de Jeanne Genoi à Condrieu. Girerd allant jusqu'à offrir huit francs d'or de dédormmagement ${ }^{77}$. L'affaire de Jehannote de Vauls à Dijon se termine également par un accord de ce type. Le texte de la renonciation de la servante à toute poursuite contre ses agresseurs se trouve placé à la suite de la longue instruction que le cas avait suscitée. Jehannote ne se rétracte pas en ce qui concerne les accusations portées et leur véracité, mais agissant - dit-elle - sur le conseil de plusieurs parents et amis tant de son côté

75. Les amendes pour viols délivrées par la cour séculière de l'archevêque, à Lyon, entre 1428 et 1442 se répartissent comme suit : plus de $5 \mathrm{lt}=11 \%$ de 3 a $5 \mathrm{lt}=$ $44,5 \%$ de 1 a $3 \mathrm{lt}=38,9 \%$ moins de $1 \mathrm{lt}=5,5 \% \mathrm{Cf}$. gonthiER, N., (1993), Délinquance, justice el société dans le Lyonnais médiéval (fin XIIT - début XVf siècles), Paris, Arguments, p. 234.

76. Cf. Leguay, J.P. «Un cas d'efforcement au Moyen Âge, le viol de Margot Simmonet", dans Mentalités, $\mathrm{n}^{\circ}$ 3, pp. 13-33.

77. Cf. Gonthicr, N., op. cit., pp. 141-142. 
que de celui des violeurs, après que les deux parties «ont traictié, pacifié et accordé ensemble de toute l'offense, injure et vilennie à [elle] faicte», elle se déclare «bien contante et aggraée» et abandonne toute plainte présente et à venir. Cette démarche permet l'élargissement (sous l'énorme caution de cinquante saluts d'or, soit soixante-quinze livres tournois !) d'André de Varley. clerc jusque là détenu pour sa participation au viol. Toutefois, l'évêque de Langres réclame la justice de ce clerc, qui n'est sans doute pas encore quitte de tracasseries ou de sanctions ${ }^{78}$.

\section{CONCLUSION}

Telle qu'elle se manifeste dans les documents consultes, la conduite des enquêtes en cas de viol met en évidence le sérieux qui caractérise la procédure, à la fin du Moyen Âge. L'attention des tribunaux pour ce type de crime traduit en outre le souci qu'a l'État de mieux discipliner les mours par le biais du pouvoir judiciaire ${ }^{79}$, tout en évitant que trop d'affaires n'échappent à sa compétence.

Le traitement de ces affaires éclaire tout particulièrement la place faite à la femme dans une société réglée par les hommes. Enjointe de porter ses plaintes à la justice, la femme ne bénéficie pas d'emblée de la confiance absolue des magistrats. Ceux-ci continuent sans doute à la voir tel un être de faible intelligence, d'une sensualité exacerbée, mobile dans ses pensées et apte au mensonge comme à la débauche. Aussi est-ce elle qu'ils examinent en premier lieu, elle de qui ils exigent des preuves de la réalité du crime. La gamme des arguments probants propose alors une image idéale de la femme : elle est innocente, étrangère aux troubles du désir, occupée seulement aux tâches que lui commande son statut de servante ou d'épouse ; elle ne cède qu'à la contrainte violente, mais non sans avoir montré un grand courage au cours de la lutte. Le texte des instructions révèle encore l'existence d'un efficace réseau d'alliances féminines s'exerçant dans le cadre de la demeure ou du quartier, pour se défendre contre le désir des hommes, le dénoncer ou secourir les victimes qu'il a détruites. L'esprit d'initiative et la force de décision des femmes s'y déploient ostensiblement. contrastant de façon frappante avec l'image d'éternelles mineures que le droit ou la religion voudraient donner d'elles.

Les procès pour viol font apparaître aussi la valeur extrême accordée à la virginité et à l'enfance. Toute corruption entreprise à l'encontre de vierges ou de fillettes n'ayant pas atteint l'âge nubile semble compromettre à la fois l'ordre matrimonial et la survie de l'espèce. On insiste sur les

78. ADCO, BII 360/01, $\mathrm{n}^{\circ} 8$, pièce $\mathrm{n}^{\circ} 3,22$ avril 1431.

79. Cet effort se poursuit en France depuis les ordonnances de saint Louis. On retrouve une analyse semblable dans A. Porteau-Bitker, article cité. a propos de la sćverité des jugements du Châtelet. 
irréparables dégradations physiques causées par le viol, sur la «nature » de la femme «cassée» et douloureuse, sur la maladie instillée en elle désormais.

Les documents judiciaires proclament enfin l'existence de l'honneur féminin, qui réside dans l'intégrité du corps, la pudeur du maintien : autant de valeurs que le viol bafoue définitivement. Les textes évoquent une «macule», une «flétrissure».

Cependant. outre l'histoire des mentalités, l'histoire sociale gagne en précision grâce à ces pièces judiciaires. On saisit davantage, à leur lecture. la situation difficile des servantes, fillettes de dix ans ou adolescentes séparées de leur famille, en butte aux passions malsaines de leur entourage, bien souvent démunies devant les dangers. Elles sont les proies les plus faciles pour les violeurs et promises a un avenir de prostitution, une fois que le viol leur a fait perdre leur «bona fama».

Le récit de ces affaires signale encore l'oisiveté des jeunes gens, leur goût pour les «fraternités», pour les bandes armées dont les jeux consistent à débusquer les filles perdues de réputation ou. à défaut d'en trouver, à chasser d'innocentes pucelles. Leurs mœurs impitoyables, leur présence tumultueuse dans les villes de la fin du Moyen Âge ont été suffisamment décrites ${ }^{80}$ pour que l'on insiste ici sur un autre aspect, moins étudié jusqu'à présent : l'attitude des témoins.

Ceux-ci sont capables d'exposer les circonstances du rapt ou du viol, de donner des détails qui confirment l'angoisse, la détresse de la victime; grâce à eux, on perçoit même les appels au secours qu'elle a lancés dans la rue, ou depuis sa chambre. Voisins et voisines de l'intéressée, ils savent aussi émettre un avis sur sa conduite passée, puisqu'ils l'ont observée et jaugée. en pratiquant ce contrôle quotidien, cet «espionnage» sans bienveillance que la disposition de l'habitat, l'étroitesse des rues, l'espace introverti du quartier rendent inévitables. Mais alors, qu'ont-ils fait quand la malheureuse appelait à l'aide ? Comment n'ont-ils pas pu. pour la plupart du moins, empêcher ou interrompre l'agression? Une légère gêne se glisse dans leur déposition lorsqu'ils expliquent qu'ils n'ont songé alors qu'à se calfeutrer dans leur logis, de peur de recevoir un mauvais coup. Cette couardise répandue en dit long sur l'atmosphère d'insécurité et de violence qui règne dans les villes au $X V^{e}$ siècle.

Ainsi, les procès pour viol viennent rappeler, à leur façon. la dureté d'un temps où s'opposent une morale rigide qui prétend régler la réputation des femmes et des maeurs masculines libres et brutales. Par la rigueur des sentences qu'elle réserve aux violeurs, la justice de la fin du Moyen Âge prétend résoudre cette contradiction sans toujours y parvenir.

80. CI. Rossiaud, J., op. cit. 